Please quote as: Bittner, E. A. C. \& Leimeister, J. M. (2015): Das TANDEM-Konzept zur Unterstützung des Wissenstransfers in altersdiversen Arbeitsgruppen. In: Exploring Demographics - Transdisziplinäre Perspektiven zur Innovationsfähigkeit im demografischen Wandel. Hrsg./Editors: Jeschke, S.; Richert, A.; Hees, F. \& Jooß, C. Verlag/Publisher: Springer Spektrum, Wiesbaden, Germany. Erscheinungsjahr/Year: 2015. Seiten/Pages: 371-382. 


\title{
Das TANDEM-Konzept zur Unterstützung des Wissenstransfers in altersdiversen Arbeitsgruppen
}

Fallstudienerkenntnisse aus der Automobilindustrie

\author{
Eva Alice Christiane Bittner, Jan Marco Leimeister ${ }^{1}$
}

Keywords: Wissensmanagement, Kollaboration, altersdiverse Arbeitsgruppen, Fallstudie

\begin{abstract}
Um eine nachhaltige Sicherung von Erfahrungswissen auch über das Ausscheiden wertvoller Erfahrungsträger hinweg zu gewährleisten, muss der Wissenstransfer zwischen Experten und Novizen im Arbeitskontext systematisch unterstützt werden. In diesem Beitrag werden Erkenntnisse aus einer Fallstudie zur Pilotierung eines Wissenstransferprozesses in einem Großunternehmen der deutschen Automobilbranche vorgestellt. In dem TANDEM-Pilotprojekt wurde ein Workshopprozess für altersgemischte Arbeitsgruppen entwickelt und erprobt, bei dem Wissen zu ausgewählten, komplexen Arbeitsprozessen durch Experten und Novizen kollaborativ in Form von Schulungsmaterialien dokumentiert wird. Die Erkenntnisse leisten einen Beitrag zur Gestaltungsforschung für wiederholbare Wissensmanagement-Gruppenprozesse. Wir zeigen auf, wie die im TANDEM-Workshopprozess eingesetzten Kollaborationstechniken dazu dienen, Herausforderungen im Wissenstransfer von Experten und Novizen zu begegnen und heterogene Arbeitsgruppen erfolgreicher zu machen. Dieser
\end{abstract}

1 Eva Alice Christiane Bittner | Universität Kassel | eva.bittner@uni-kassel.de

Jan Marco Leimeister | Universität Kassel, Universität St. Gallen (HSG) | leimeister@unikassel.de

S. Jeschke et al. (Hrsg.), Exploring Demographics, DOI 10.1007/978-3-658-08791-3_35,

(C) Springer Fachmedien Wiesbaden 2015 
Beitrag gibt Praktikern einen Musterprozess zur Unterstützung des Wissenstransfers in altersdiversen Arbeitsgruppen an die Hand.

\section{$1 \quad$ Einführung}

Wissen ist in einem immer schneller wandelnden Umfeld zur zentralen erfolgskritischen Ressource für viele Organisationen geworden (Sawhney und Prandelli 2000: 26; Madhoushi und Sadati 2010: 392). Das notwendige Wissen für Arbeitsprozesse und Innovationen wird durch technologischen Fortschritt immer vielfältiger und komplexer (DeLong 2004: 16). Oft hängt der erfolgreiche Betrieb technologiegetriebener Arbeitsprozesse maßgeblich von der Expertise einzelner Wissensträger ab. Verlassen diese Personen das Unternehmen oder stehen aus anderen Gründen nicht zur Verfügung, sind diese Prozesse gefährdet. Nur wenn es gelingt, Wissen in der Organisation zu bewahren, Wissensaustausch zwischen Mitarbeitern zu unterstützen und systematisch neues Wissen zu generieren, bleiben Organisationen innovations- und wettbewerbsfähig.

Andererseits hat sich die demographische Struktur der Belegschaft in vielen Branchen stark verändert und wird dies in den kommenden Jahrzehnten in zunehmendem Maße weiter tun. Die erwerbstätige Bevölkerung in vielen Industrienationen wird älter und kleiner, wenn sich die Generation der „Babyboomer“ dem Rentenalter nähert. Die viel kleinere Gruppe der „Generation Y“ muss innerhalb kurzer Zeit befähigt werden, Ihre Expertise und Aufgaben zu übernehmen. So ist beispielsweise in Deutschland zwischen 2000 und 2025 ein Rückgang der erwerbstätigen Bevölkerung um 20\% zu erwarten. Demgegenüber steht ein erwartetes Wachstum des Rentneranteils um 50\% (DeLong 2004: 14). Lebenslange Erfahrung kann jedoch kaum innerhalb weniger Ausbildungsjahre erlernt und nur schwer von Mensch zu Mensch weitergegeben werden (Sporket 2011: 55-57).

Ungeteiltes Wissen kann zum Hindernis in der Zusammenarbeit von Arbeitsgruppen werden und stellt eine wichtige Herausforderung für Designer von Kollaborationsprozessen dar (Piirainen et al. 2012: 1092; Leimeister 2014: 142). In Organisationen mit heterogenen Belegschaften müssen Wissensmanagementprozesse etabliert werden, um junge, weniger erfahrene Mitarbeiter frühzeitig darauf vorzubereiten, komplexe Arbeitsprozesse selbständig durchzuführen und Expertenwissen ihrer erfahrenen Kollegen zu übernehmen. Bisher herrscht jedoch in Forschung und Praxis ein Mangel an Wissen, wie die Wissenslücke zwischen Experten und Novizen in heterogenen Arbeitsgruppen systematisch überbrückt werden kann. 
Dieser Beitrag nimmt sich dieser Forschungslücke an, indem er einen Lösungsansatz in Form eines Workshopprozessdesigns zur Unterstützung des Wissenstransfers zwischen Experten und Novizen vorstellt. In einer realweltlichen Fallstudie wurde der Workshopprozess mit 48 altersdiversen Facharbeitern in einem Automobilkonzern pilotiert. Wir stellen Ergebnisse zu folgenden Leitfragen vor: Inwiefern unterstützt der TANDEM-Workshopprozess den Wissenstransfer in altersdiversen Arbeitsgruppen? Welche Herausforderungen entstehen in den neu gebildeten Gruppen in der Fallstudie? Wie werden diese gelöst?

\section{Das TANDEM-Workshopkonzept}

Die Zielsetzung des TANDEM-Workshopprozesses liegt darin, Teams aus je 3 erfahrenen und 3 unerfahrenen Teilnehmern bei der Dokumentation eines Arbeitsprozesses in Form von Schulungsbausteinen zu unterstützen und währenddessen den Wissenstransfer zwischen den Teilnehmern anzuregen. Jedes Team durchläuft eine dreitägige Workshopserie aus je einem Kick-off-, Ausarbeitungs- und Finalisierungsworkshop in Abständen von jeweils ca. 2 bis 4 Wochen.

\subsection{Kick-off-Workshop}

Der erste Workshop hat zum Ziel, eine Struktur für einen Schulungsbaustein zu entwickeln und festzuhalten, was man bei jedem Arbeitsschritt in dem ausgewählten Arbeitsprozess wissen und können muss. Der Kick-off-Workshop hat folgende Struktur, die aus vier Phasen besteht (vgl. Abb. 1).

Erst allein, dann in erfahrungsgemischten TANDEM-Paaren und schließlich in der Gruppe entwickeln die Teilnehmer zunächst eine Grobstruktur des zu dokumentierenden Arbeitsprozesses.

In der ersten Phase des Workshops sollen die Teilnehmer alleine auf Kärtchen den Arbeitsverlauf notieren, ohne die Schritte mit den Teampartnern zu diskutieren oder die Reihenfolge zu besprechen. Der beteiligte Forscher, der den Workshopprozess moderiert, unterstützt Teilnehmer, die Probleme haben, den Arbeitsprozess zu strukturieren oder aufzuschreiben.

Anschließend kommt die im Rahmen des TANDEM-Projekts entwickelte MindMerger Technik zur Entwicklung eines gemeinsamen Verständnisses zum Einsatz (Bittner und Leimeister 2014: 122). In der zweiten Phase des Workshops führen jeweils zwei Teilnehmer (ein erfahrener und ein unerfahrener) ihre Vorschläge in einem gemeinsamen Entwurf des Arbeitsprozesses zusammen. 


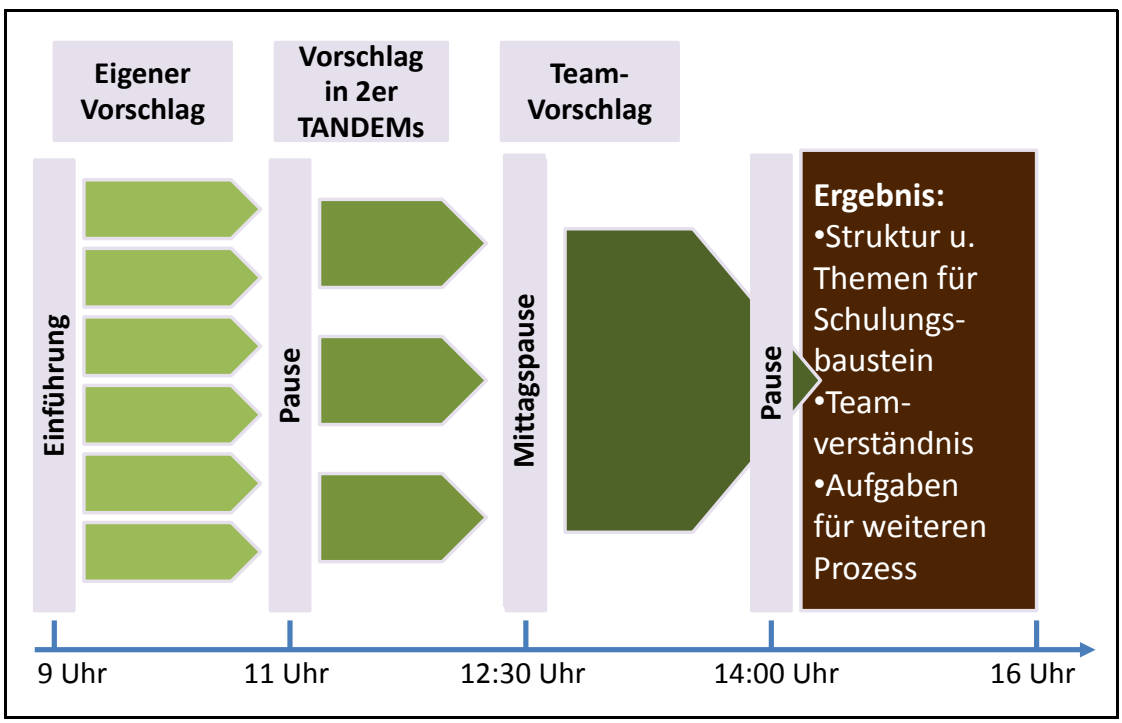

Abb. 1: Aufbau der ersten Workshops

Die Teilnehmer tauschen zunächst ihre Karteikarten aus der individuellen Arbeitsphase aus und hängen die Karten mit den Arbeitsschritten untereinander auf. Im folgenden Arbeitsauftrag sollen die Tandem-Partner sich die Arbeitsprozessdokumentation kritisch ansehen. Die Stellen, an denen die Teilnehmer ihrem Partner Verständnisfragen stellen wollen, werden markiert und anschließend geklärt. Neue Aspekte, die in diesem Gespräch entstanden sind, werden ergänzt, um die individuellen Prozessbeschreibungen klarer zu machen. Im nächsten Schritt vergleichen die Tandem-Partner ihre Arbeitsabläufe und erkennen Unterschiede zwischen ihrer und der Dokumentation des Partners. Aspekte, die nur in einem der Vorschläge auftauchen und übernommen werden sollen, wenn die Arbeitsabläufe zusammengeführt werden, sowie Wiedersprüche in den Prozessbeschreibungen werden mit farbigen Punkten markiert. Kärtchen, die als unterschiedlich markiert sind, werden in die zusammengeführte Version übernommen, da sie jeweils nur einmal vorhanden, aber wichtig für den Prozessablauf sind. Bei Kärtchen mit Konflikten muss das TANDEM entscheiden, auf welche Sichtweise sich die Partner einigen und welche Version sie in den gemeinsamen Vorschlag übernehmen. Ihren zusammengeführten Prozessvorschlag stellen die Teilnehmer den anderen TANDEMs vor.

In der letzten Phase des Kick-off-Workshops erarbeiten alle sechs Teilnehmer gemeinsam einen Teamvorschlag. Nach der Vorstellung wird über die 
einzelnen TANDEM-Vorschläge diskutiert. Die Aspekte, die unverständlich sind, werden geklärt. Die Vorschläge werden miteinander verglichen. Abweichende Meinungen zu Abfolge und Inhalt des Prozesses werden diskutiert. Die Kärtchen aus unterschiedlichen Vorschlägen werden in eine gemeinsame Version zusammengeführt. Dabei werden Kärtchen außer Acht gelassen, falls sie zu den übernommenen nahezu identisch sind oder sich während der Diskussion als überflüssig erweisen. Außerdem werden einige neue Kärtchen geschrieben, die im Gespräch neu identifizierten Aspekte aufnehmen. Zuletzt sammeln alle Teilnehmer in einem Brainstorming ihre Ideen dazu, was ein Mitarbeiter wissen und können muss, um den einzelnen Arbeitsschritt zu bearbeiten; welche Probleme in den Arbeitsschritten auftreten können; und in welchen anderen Bereichen ähnliches Wissen und Können auch benötigt wird.

Am Ende des ersten Workshops stehen die Themen und eine Struktur für den Schulungsbaustein fest. Die Teilnehmer bekommen einen Arbeitsauftrag bis zum nächsten Workshop: Jeder Teilnehmer soll mit seinem TANDEMPartner am Arbeitsplatz durchspielen, wie er einem neuen Mitarbeiter die Lerninhalte beibringen würde und den Prozess mit Fotos, einer Beschreibung etc. dokumentieren.

\subsection{Ausarbeitungsworkshop}

Das Ziel des Ausarbeitungsworkshops liegt darin, dass eine klare Beschreibung jedes einzelnen Arbeitsschrittes im Arbeitsprozess in Form eines Lerngesprächs mit Bildmaterial erstellt wird. Der Workshop wurde genau wie der Kick-offWorkshop in vier Phasen eingeteilt (vgl. Abb. 2).

Zunächst sollen die Teilnehmer den Arbeitsprozess überprüfen und mit Bildern ergänzen, die Teilnehmer während der Hausaufgabe gemacht hatten. Der Moderator des Workshops prüft hierfür zusammen mit den Teilnehmern alle Prozessschritte, indem er sie vorliest.

In der zweiten Phase des Workshops fertigen die Teilnehmer eine eigene Beschreibung der Arbeitsschritte an. Für diese Aufgabe erhalten alle Teilnehmer Laptops mit dem Gruppenunterstützungssystem thinkTank 3 (GroupSystems) und eine Einführung in das System. Das Gruppenunterstützungssystem ermöglicht es, Inhalte, die die Teilnehmer generieren, in Echtzeit für alle zu aktualisieren. Alle Prozessschritte werden zwischen den Teilnehmern zur Bearbeitung aufgeteilt und die Teilnehmer notieren stichpunktartig, was im jeweiligen Schritt zu wissen und zu können ist. Wenn ein Teilnehmer mit seinen Prozessschritten komplett fertig ist, kann er die weiteren Schritte, die einem anderen Teilnehmer zugeteilt wurden, ergänzen. Durch dieses Vorgehen entsteht in relativ kurzer Zeit für jeden Prozessschritt ein Handzettel mit den Kernthemen für die Ausarbeitung. 


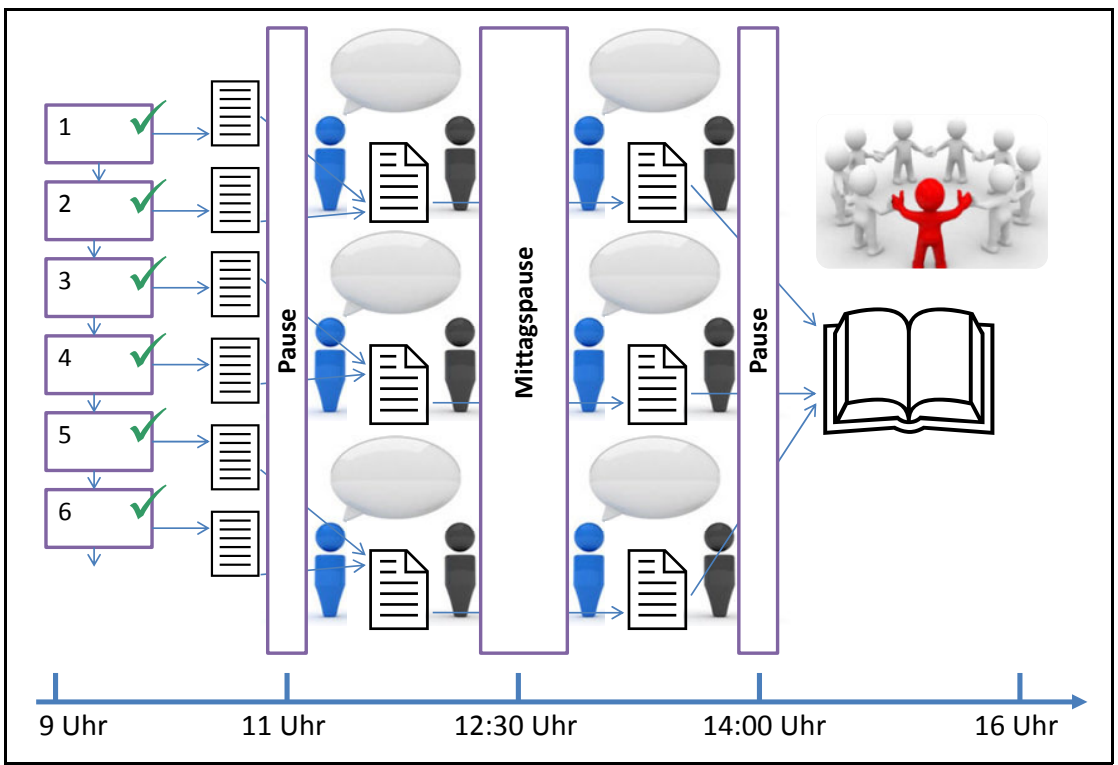

Abb. 2: Aufbau der Ausarbeitungsworkshops

In der dritten Phase findet die Entwicklung der Lerngespräche statt. Die Gruppe wird hierzu in drei TANDEMs mit jeweils zwei Personen (einem erfahrenen und einem unerfahrenen Mitarbeiter) geteilt. Der Dialog wird chat-ähnlich im Gruppenunterstützungssystem erstellt. Jeweils ein TANDEM-Partner soll dem anderen den Arbeitsschritt erklären. Der andere hat die Aufgabe, kritische Fragen zur Erklärung zu stellen. Wo sinnvoll, werden Bilder zu den Erklärungen hinzugefügt oder eigene Skizzen angefertigt.

Abb. 3 zeigt beispielhaft die Fragen-Antwort-Logik des Lerndialogs. 


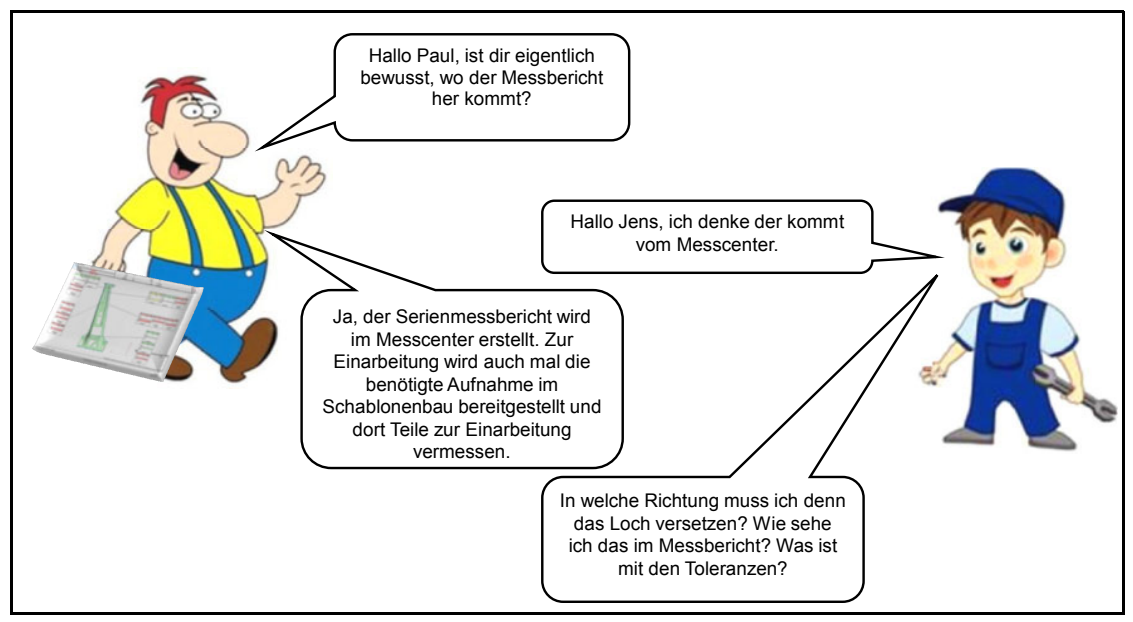

Abb. 3: Beispiel aus dem Lerndialog

In der vierten Phase werden die Lerngespräche zusammengeführt. Jedes TANDEM trägt seinen Lerndialog vor. Die Zuhörer kommentieren und ergänzen. Lücken, die nicht direkt vor Ort geschlossen werden können (z.B. fehlende Bilder oder Anleitungen), werden als Arbeitsauftrag festgehalten, den die Teilnehmer wieder als Hausaufgabe bearbeiten müssen. Zusätzlich werden die Teilnehmer gebeten, den Entwurf des Lerndialogs mit anderen Mitarbeitern im Betrieb zu erproben und Feedback dazu einzuholen.

\subsection{Finalisierungsworkshop}

Das Ziel des letzten Workshops ist es, das Lerngespräch mit weiterem Bildmaterial für alle Arbeitsschritte anzureichern, Übungs- und Testaufgaben zum Schulungsbaustein zu entwickeln und Ideen zum Einsatz der Schulungsmaterialien, zur Verbesserung des Workshopkonzepts und des dokumentierten Arbeitsprozesses zu sammeln.

Zuerst wird der Lerndialog überprüft. Alle von den Teilnehmern vorgeschlagenen Anmerkungen werden durchgegangen und der Lerndialog wird vervollständigt. In der zweiten Phase werden von den TANDEMs Übungsaufgaben und ein Test zur Selbstkontrolle für neue Mitarbeiter erstellt. Gegenseitig wird geprüft, ob die Aufgaben der anderen TANDEMs klar formuliert, nicht zu leicht und nicht zu schwer für einen neuen Mitarbeiter sind. Desweiteren wird Feedback von den Teilnehmern eingeholt, wie die Schulungsbausteine bei ihrer Arbeit eingesetzt werden könnten und was im Unternehmen gegeben sein müsste, damit sie den Schulungsbaustein gut nutzen könnten. Außerdem sollen 
die Teilnehmer überlegen, wie die Wissensweitergabe in ihrem Bereich sonst noch verbessert werden könnte.

\section{Fallstudienbeschreibung}

Das TANDEM-Workshopkonzept wurde in einem großen Unternehmen der Automobilindustrie pilotiert. Wie viele andere Firmen, steht auch dieses Unternehmen vor der Herausforderung, seine Mitarbeiter zu einem Wissenstransfer zu unterschiedlichen Themen anzuregen. Arbeitnehmer, die eine lange Zeit im Betrieb tätig sind, haben große Erfahrung und ein tiefes Verständnis für die Arbeitsabläufe. Gleichzeitig sind sie mit dem stets wachsenden Tempo technologischer Veränderungen in ihrem Arbeitsumfeld konfrontiert. Das Unternehmen läuft Gefahr, Kenntnisse und implizites Wissen der Mitarbeiter, die sich dem Rentenalter nähern, zu verlieren, wenn die erforderlichen Maßnahmen zur Unterstützung des Wissenstransfers zu den nachfolgenden Mitarbeitern nicht ergriffen werden. Andererseits bringen neue Arbeitnehmer die aktuellste technische Ausbildung mit sich und können innovative Lösungen in die bestehenden Arbeitsprozesse einbringen. Dennoch fehlen ihnen das spezifische Wissen und die Erfahrung bei komplexen Aufgaben. Junge Mitarbeiter, die erst kürzlich ihre Berufsausbildung abgeschlossen haben und ältere Mitarbeiter, die mehr Erfahrung besitzen, sollen voneinander lernen, um das kritische Wissen vor dem Verschwinden aus der Organisation zu bewahren. Die Pilotierung des Kollaborationsprozesses hatte zum Ziel, in einer Reihe von Workshops die langjährige Erfahrung altersdiverser Werkzeugbauer zu integrieren. Die Facharbeiter wurden von einem erfahrenen Moderator durch den gesamten Prozess geführt, um Schulungsbausteine für ausgewählte Arbeitsabläufe in ihrem Arbeitsumfeld $\mathrm{zu}$ erarbeiten. Diese Schulungsmaterialien sollen unerfahrenen Mitarbeitern helfen, komplexe Arbeitsaufgaben selbständig auszuführen.

Die Heterogenität der Gruppenmitglieder, die für das Projekt gewonnen wurden, zeigt sich in mehreren Dimension: Alter, Geschlecht, Ausbildung, Arbeitserfahrung, Unternehmenszugehörigkeit. Jede Gruppe setzt sich aus jeweils drei erfahrenen Experten und drei unerfahrenen Novizen im Hinblick auf das als Gruppe zu dokumentierende Thema zusammen. Insgesamt haben 48 Facharbeiter an dem Projekt teilgenommen, fünf Frauen und 43 Männer. Das Durchschnittsalter von erfahrenen bzw. unerfahrenen Teilnehmern betrug jeweils 44,16 und 24,09 Jahre, dabei war der älteste Teilnehmer 57 und der jüngste 19 Jahre alt. Die gesamte Arbeitserfahrung der Teilnehmer wies eine hohe Bandbreite von fünf Wochen bis zu 42 Jahren auf. 
Das Pilotierungsprojekt wurde von zwei Forschern beobachtend begleitet. Ergänzend wurden Interviews mit Teilnehmern durchgeführt, die entstandenen Dokumente ausgewertet, sowie vor und nach jedem Workshop Selbsteinschätzungen der Teilnehmer in Form von Fragenbögen erhoben. Eine ausführliche Darstellung der Evaluation in Hinblick auf Wissenstransfer und gemeinsames Verständnis findet sich in Bittner et al. (2013) und Bittner und Leimeister (2014).

\section{Erkenntnisse aus der Pilotierung für die Wissenstransferforschung und -praxis}

Im Folgenden werden zentrale Erkenntnisse der qualitativen Fallstudienanalyse zusammenfassend dargestellt.

\subsection{Individuelle Arbeitsphase essentiell zur Explizierung des Erfahrungswissens}

Die individuellen Beschreibungen der Arbeitsabläufe waren oft sehr unterschiedlich, obwohl alle Teilnehmer einer Gruppe denselben Arbeitsprozess beschrieben haben. Die Beschreibung des Arbeitsprozesses fiel nicht allen leicht. Insbesondere Experten fühlten sich oft mit einem Zustand ,unbewusster Kompetenz" konfrontiert (Ambrose et al. 2010: 97). Auf Grund ihrer langjährigen Erfahrung, fiel es ihnen schwer, Arbeitsprozesse, die sie in der Praxis routiniert und ohne nachzudenken durchführen, schriftlich festzuhalten. Desweiteren äußerten viele Experten zu Beginn der Zusammenarbeit Unsicherheit über den Wissensstand der unerfahrenen Kollegen und darüber, auf welchem Level der Schulungsbaustein aufbauen musste. Für beide Herausforderungen zeigte sich die individuelle Arbeitsphase zu Beginn des ersten Workshops als geeigneter Lösungsansatz. Experten waren dadurch gefordert, ihr abstraktes Wissen zu strukturieren und dokumentieren. Novizen legten ihr Verständnis des Arbeitsprozesses offen, ohne sich in einer Gruppe verstecken zu können. Die individuellen Dokumente dienten im späteren Prozess als „boundary objects“ zur Entwicklung eines gemeinsamen Verständnisses (Bittner und Leimeister 2014: 123).

\subsection{Einsatz der MindMerger Kollaborationstechnik verbessert gemeinsames Verständnis zwischen Experten und Novizen}

Die iterative Zusammenführung der Arbeitsprozessstruktur von individuellem über paarweisem zur Gruppen-Entwurf erwies sie als vorteilhaft für die Entwicklung eines gemeinsamen Verständnisses zwischen Experten und Novizen 
(Bittner und Leimeister 2014: 132). Insbesondere fiel auf, dass durch die paarweise Interaktion auch zurückhaltende, unerfahrene Teilnehmer zur Beteiligung angeregt wurden. Durch das iterative Vorgehen wurden alle Teilnehmer eingebunden. Die Arbeit mit den individuellen und paarweisen Arbeitsprozessdokumenten führte dazu, dass Unterschiede und Widersprüche in den mentalen Modellen systematisch aufgedeckt werden konnten. In den Experten-NovizenTANDEMs wurde dabei hauptsächlich das Verständnis der unerfahrenen TANDEM-Kollegen erweitert und erfahrene Mitarbeiter wurden auf Arbeitsschritte hingewiesen, die ihnen vorher unbewusst waren. Im Gegensatz dazu wurde während der Zusammenführung in ein Gruppendokument hauptsächlich aufgedeckt, wo Experten über die Jahre unterschiedliche Vorgehensweisen entwickelt hatten. Durch die Arbeit an gemeinsamem Material konnte ein Konsens ausgearbeitet werden, der als Grundlage für die Ausarbeitung im zweiten und dritten Workshop diente.

\subsection{IT-gestützte Fragen-und-Antwort-Technik nutzt Potentiale von Experten-Novizen-Interaktion}

Als weitere zentrale Phase im Workshopprozess für den Wissenstransfer hat sich die kollaborative Erstellung eines Lerndialogs durch altersdiverse TANDEMs unter Nutzung des Gruppenunterstützungssystems thinkTank herausgestellt. In dieser Phase konnte zum einen der direkte Wissenstransfer zwischen den Beteiligten unterstützt werden, indem unerfahrene Mitarbeiter genau die Fragen beantwortet bekamen, die ihnen auf ihrem Wissenstand zur Durchführung des Arbeitsprozesses halfen. Zum anderen wurde das tazite Erfahrungswissen der Teilnehmer unmittelbar dokumentiert und für weitere Mitarbeiter nutzbar gemacht. Teilnehmer signalisierten, dass sie sich mit den entstandenen Lernmaterialien viel besser identifizieren können, als mit bisher verfügbaren, von externen Beratern erstellten Schulungsunterlagen. Desweiteren äußerten vor allem unerfahrene Teilnehmer, dass sie nach der Lerndialogerstellung viel selbstsicherer waren, welchen Beitrag sie für die Gruppenarbeit leisten konnten. Novizen wurden bestärkt, indem deutlich wurde, dass ihre Fragen ein notwendiger Bestandteil für das Funktionieren des Lerndialogs sind. Außerdem ermöglichte die IT-gestützte Zusammenarbeit einen anonymisierten Austausch auf Basis der Inhalte, unabhängig vom Ersteller des einzelnen Beitrags. Durch das gemeinsame Gruppenziel wurden so evtl. existierende Vorurteile und Hemmungen reduziert und die Teilnehmer berichteten einen gesteigerten Gruppenzusammenhalt und mehr geteiltes Wissen nach dem Workshop. 


\section{Fazit/Ausblick}

Dieser Beitrag präsentiert den TANDEM-Workshopprozess als Lösungsansatz für die Herausforderungen des Wissenstransfers zwischen erfahrenen und unerfahrenen Mitarbeitern im demografischen Wandel. Neben der Wissensdokumentation im erstellten Schulungsbaustein für andere Mitarbeiter liegt ein Hauptziel des Projektes in der direkten Weitergabe von implizitem Erfahrungswissen zwischen den Teilnehmern. Außerdem zielt der Prozess darauf ab, dass Barrieren zwischen erfahrenen und unerfahrenen Kollegen abgebaut werden und Strukturen entstehen, die einen verbesserten Wissensaustausch im Arbeitsalltag begünstigen. Erfahrene Mitarbeiter profitieren von der Teilnahme am TANDEM@VW-Projekt durch verstärkte Wertschätzung für ihre Expertise sowie neue Impulse für ihre eigene Arbeit aus den frischen Perspektiven neuer Kollegen. Außerdem erhalten sie aus dem Prozess entstehende Schulungsmaterialien, die sie bei ihren Einarbeitungsaufgaben für neue Kollegen einsetzen können. Unerfahrene Mitarbeiter bekommen in geschütztem Rahmen außerhalb des Arbeitsalltags die Möglichkeit, von Experten zu lernen, Fragen zu Arbeitsprozessen zu stellen, die sie in Zukunft meistern müssen, und ihr eigenes Wissen zu erweitern. Durch die Kombination verschiedener Kollaborationsmechanismen, insbesondere die Verbindung von individuellen und kollaborativen Phasen, den Einsatz der MindMerger Technik für Shared Understanding und IT-gestützte Dialogerstellung kann der TANDEM-Workshopprozess den Wissenstransfer verbessern. Designern von Kollaborationsprozessen wird mit dem TANDEM-Prozess ein Lösungsansatz für Wissenstransfer-Dienstleistungen (Leimeister 2012: 22) an die Hand gegeben, der die Entwicklung von gemeinsamem Verständnis und den Wissenstransfer in heterogenen Arbeitsgruppen verbessern und die Teameffektivität steigern kann.

\section{Literatur}

Ambrose, Susan A.; Bridges, Michael W.; DiPietro, Michele; Lovett, Marsha C.; Norman, Marie K. (2010): How learning works: Seven research-based principles for smart teaching. JosseyBass, San Francisco.

Bittner, Eva Alice Christiane; Hoffmann, Axel; Leimeister, Jan Marco (2013): Engineering for Shared Understanding in Heterogeneous Work Groups - An Action Research study at a German Automotive Company. $13^{\text {th }}$ annual meeting on Group Decision and Negotiation. Stockholm.

Bittner, Eva Alice Christiane; Leimeister, Jan Marco (2014): Creating Shared Understanding in heterogeneous work groups - Why it matters and how to achieve it. In: Journal of Management Information Systems, 31 (1), S. 111-143. 
DeLong, David W. (2004): Lost Knowledge: Confronting the Threat of an Aging Workforce. Oxford University Press, Oxford.

Leimeister, Jan Marco (2012): Dienstleistungsengineering und -management. Springer Gabler, Berlin, Heidelberg.

Leimeister, Jan Marco (2014): Collaboration Engineering. IT-gestützte Zusammenarbeitsprozesse systematisch entwickeln und durchführen. Springer Gabler, Berlin, Heidelberg.

Madhoushi, Mehrdad; Sadati, Abdolrahim (2010): Knowledge management, antecedent of organizational innovation and competitiveness. In: Proceedings of the European Conference on Intellectual Capital. Lisbon, Portugal, S. 391-398.

Piirainen, Kalle A.; Kolfschoten, Gwendolyn L.; Lukosch, Stephan (2012): The Joint Struggle of Complex Engineering: A Study of the Challenges of Collaborative Design. In: International Journal of Information Technology \& Decision Making, 11 (6), S. 1087-1125.

Sawhney, Mohanbir ; Prandelli, Emanuela (2000): Communities of creation: Managing distributed innovation in turbulent markets. In: Management Review, 42 (4), S. 24-54.

Sporket, Mirko (2011): Organisationen im demographischen Wandel: Alternsmanagement in der betrieblichen Praxis. Springer, Wiesbaden. 